Design and Characterization of Photoelectrodes from First Principles

T. Ogitsu, B. Wood, W. Choi, M. Huda, S. Wei

May 14, 2012 
This document was prepared as an account of work sponsored by an agency of the United States government. Neither the United States government nor Lawrence Livermore National Security, LLC, nor any of their employees makes any warranty, expressed or implied, or assumes any legal liability or responsibility for the accuracy, completeness, or usefulness of any information, apparatus, product, or process disclosed, or represents that its use would not infringe privately owned rights. Reference herein to any specific commercial product, process, or service by trade name, trademark, manufacturer, or otherwise does not necessarily constitute or imply its endorsement, recommendation, or favoring by the United States government or Lawrence Livermore National Security, LLC. The views and opinions of authors expressed herein do not necessarily state or reflect those of the United States government or Lawrence Livermore National Security, LLC, and shall not be used for advertising or product endorsement purposes.

This work performed under the auspices of the U.S. Department of Energy by Lawrence Livermore National Laboratory under Contract DE-AC52-07NA27344. 


\title{
Design and Characterization of Photoelectrodes from First Principles
}

\section{${ }^{1}$ Tadashi Ogitsu, ${ }^{1}$ Brandon Wood, ${ }^{1}$ Wooni Choi, ${ }^{2}$ Muhammad Huda, and ${ }^{3}$ Suhuai Wei}

\author{
${ }^{1}$ Lawrence Livermore National Laboratory \\ ${ }^{2}$ University of Texas, Arlington \\ ${ }^{3}$ National Renewable Energy Laboratory
}

\begin{abstract}
Although significant performance improvements have been realized since the first demonstration of sunlight-driven water splitting in 1972, mainstream adoption of photoelectrochemical (PEC) cells remains limited by an absence of cost-effective electrodes that show simultaneously high conversion efficiency and good durability. Here we outline current and future efforts to use advanced theoretical techniques to guide the development of a durable, high-performance PEC electrode material. Working in close collaboration with experimental synthesis and characterization teams, we use a twofold approach focusing on: 1) rational design of novel high-performance electrode materials; and 2) characterization and optimization of the electrode-electrolyte interface.
\end{abstract}

\section{Introduction}

A photoelectrochemical (PEC) hydrogen production device uses sunlight and water to generate hydrogen gas with no adverse emissions, and as such is considered an ideal sustainable energy solution. Since the first successful demonstration of hydrogen production from sunlight and water using a $\mathrm{TiO}_{2}$ photoelectrode in 1972,1 steady improvement on solar-to-hydrogen (STH) efficiency has been made. For instance, in 19971998, a silicon triple-junction solar harvester combined with cobalt-based co-catalyst resulted in a STH efficiency of about $8 \% .^{2,3}$ In 1998, the current record STH efficiency of $12.4 \%$ was established at NREL using a GaInP $2 / \mathrm{GaAs}$ tandem cell with a Pt co-catalyst. ${ }^{4}$

Despite these breakthroughs, the PEC research community has faced great challenges in achieving high STH and durability simultaneously, which has impeded commercial use of PEC technology. Since no known material currently satisfies established U. S. Deparment of Energy target windows that would lead to widespread market adoption, ${ }^{5-11}$ it is highly desirable to devise a targeted, rational approach for developing entirely new photoelectrode materials and/or surface modifications. This has proven challenging, in part because it is not fundamentally understood how the interplay between the various electrode materials properties impacts the overall device performance. In this regard, advanced theoretical techniques are ideally suited for offering a detailed 
description of the underlying properties, and as such could be an extremely valuable tool for intelligently guiding future design efforts.

In practice, many of the materials properties that directly impact solar harvesting efficiency, corrosion resistance, and catalytic activity appear to be correlated. As a result, optimizing one property (e.g., STH efficiency) frequently compromises another (e.g., durability). One of the challenges has been to understand the nature of this correlation in the bulk electrode materials, and whether key properties can be simultaneously optimized in real devices. Accordingly, our first approach focuses on the use of first-principles density functional theory (DFT) calculations to investigate mechanisms for independently improving intrinsic semiconductor properties to achieve improved performance. The understanding provided by these studies has led to consistent modification of PEC material design strategies over the last decade (see Fig. 1).12-33
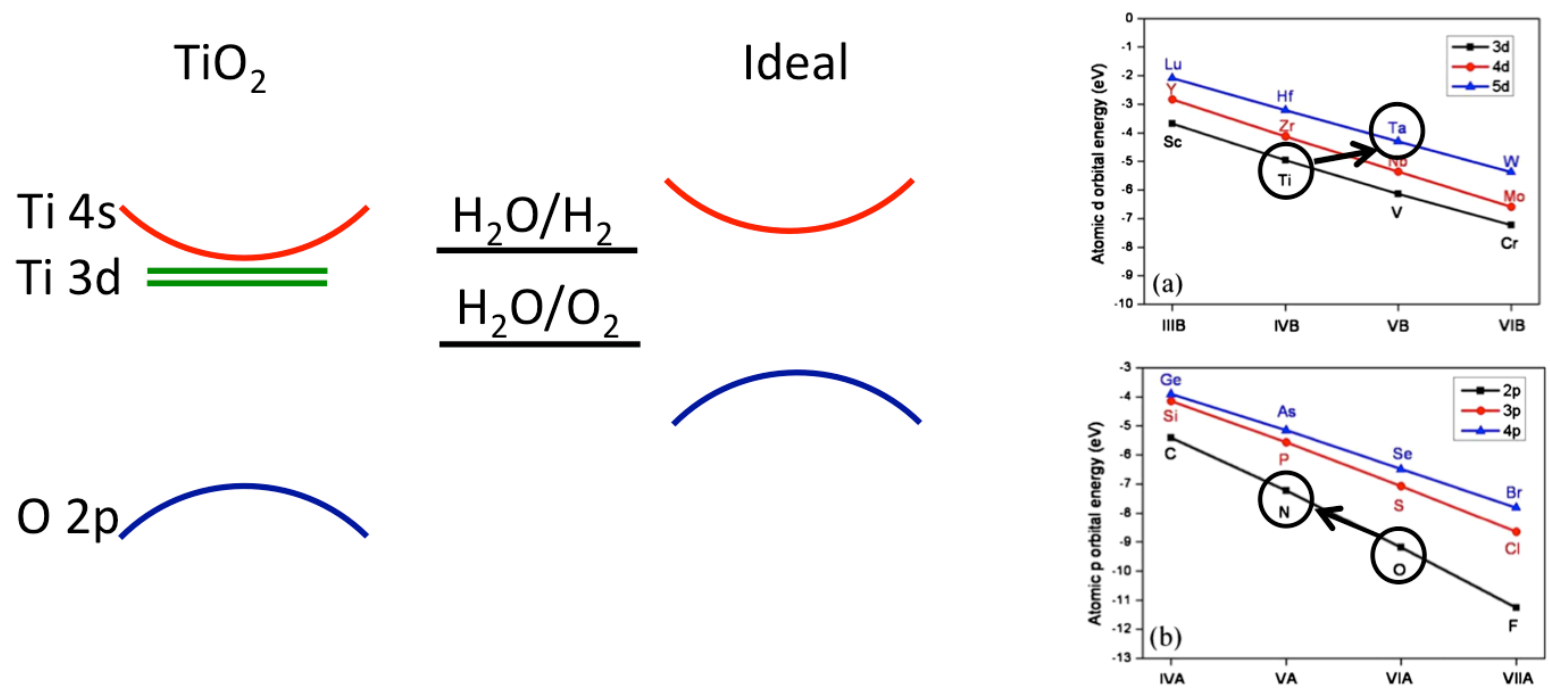

Figure 1: Cartoon images describing one possible strategy for improving $\mathrm{TiO}_{2}$ electrode properties. ${ }^{23}$ From left to right: Schematic electronic structure of $\left.\mathrm{TiO}_{2} ; 6\right) \mathrm{H}_{2} / \mathrm{O}_{2}$ water redox potentials; schematic band structure of an ideal PEC electrode; plots on levels of atomic orbitals (from ref [23]). $\mathrm{TiO}_{2}$ has a conduction band (CB) that is too low with respect to the water redox potentials and possesses localized character owing to the Ti $3 d$ states; similarly, the $02 p$-derived valence band (VB) is too low and localized. An ideal material should have $\mathrm{CB}$ and VB edges straddling the $\mathrm{H}_{2} / \mathrm{O}_{2}$ redox potentials so as to overcome any electron-transfer reaction barriers with minimal energy loss, and possessing delocalized character to ensure good carrier transport. The right-hand plot shows that one can rationally substitute atoms in order to improve the band alignment and the transport, for instance by substituting Ti with Ta (or W) and 0 with N.23

A second challenging aspect of this problem is the lack of information on the microscopic properties of electrode-electrolyte interface. When the electrode is immersed in electrolyte (even without illumination), the surface becomes contaminated by foreign chemical species. When the electrode is illuminated, the situation becomes even more complex. In addition to hydrogen and oxygen evolution, various additional types of chemical reactions, including photocorrosion, can be driven at the electrode-electrolyte interface by photogenerated carriers. Without detailed information on the microscopic structure and chemistry of the interface, formulating a consistent strategy to optimize interfacial properties becomes extremely difficult, if not impossible. In order to better 
understand the active interfacial processes, we have begun performing large-scale firstprinciples molecular dynamics simulations to examine the structure and reactivity of realistic electrode-electrolyte interfaces (see Fig 2). ${ }^{29-33}$ Such simulations have become possible only in recent years, thanks to significant advances in supercomputer performance and novel software algorithms

To summarize, our theory effort focuses on two areas: electrode material design and characterization of interfacial properties. Dr. Wei and Dr. Huda will refine and deploy the materials design strategies that have been developed in the past several years in order to screen and optimize candidate electrode materials. The LLNL team will continue their theoretical studies on the semiconductor-electrolyte interface using first-principles molecular dynamics simulations. Their focus will be on investigating surface stabilization and activation mechanisms based on modification of the interfacial structure and chemistry. In all cases, information obtained from these activities will be shared with the collaborators on the materials synthesis and characterization teams, as well as the wider PEC research community, in order to accelerate the development of a viable PEC electrode material.

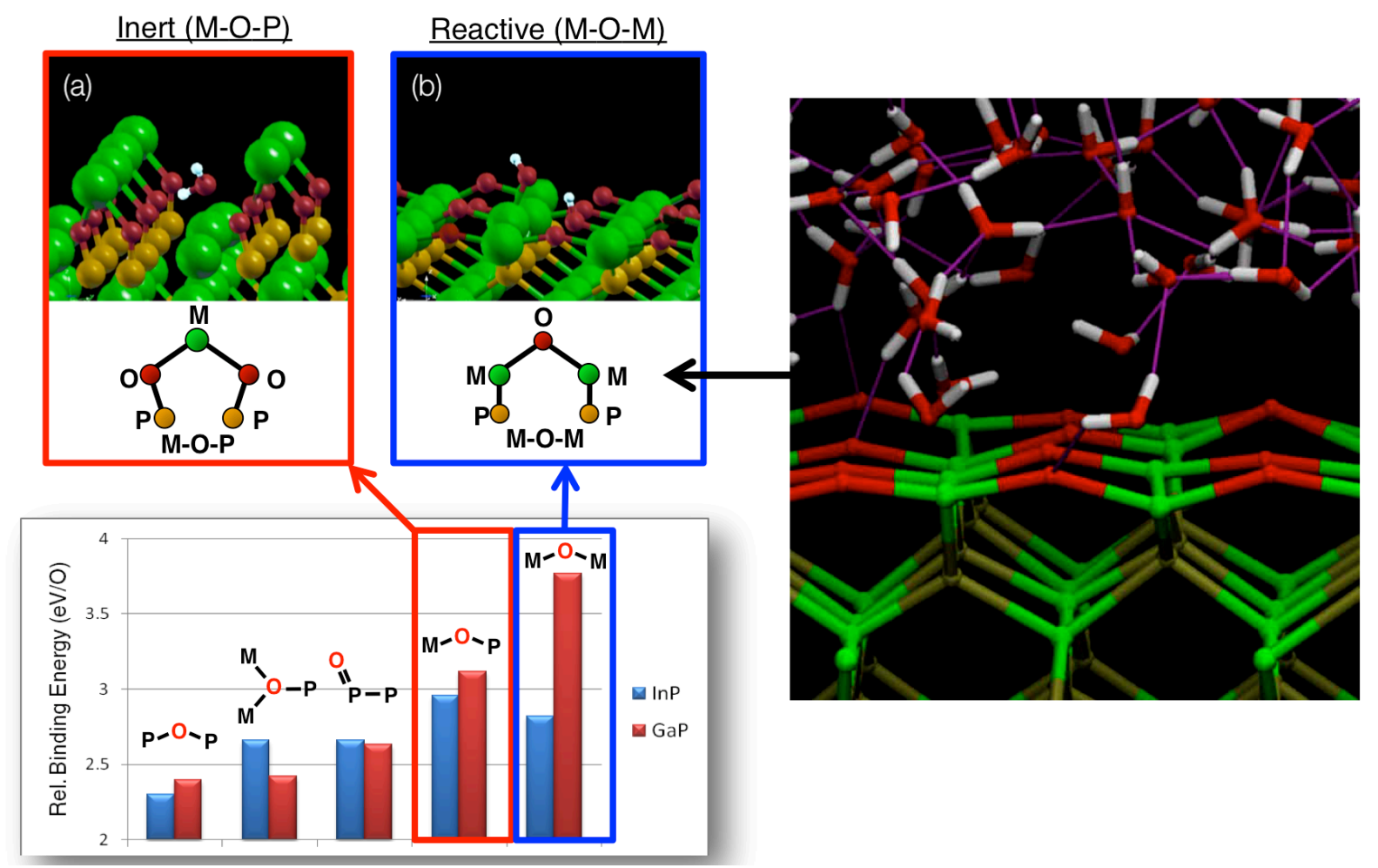

Figure 2: It was demonstrated that the local bonding topologies of an oxidized III-V surface are a descriptor of chemical activity (left). This local model approach was successfully applied to rationalize the chemical activities observed in the direct first-principles molecular dynamics simulations of the water/III-V interface (right). From ECS presentation, Boston (2011) by Wood, Schwegler, and Ogitsu. 


\section{Task I: Rational Design of Electrode Materials}

Focus: Assessment and simultaneous optimization of electronic, optical, and transport properties of crystalline and nano-crystalline photocatalyst materials.

The main objective is to theoretically/computationally investigate the electronic structures of novel photocatalysts materials, and to calculate their optical gaps and transport properties. The goal is to tune these properties to match the $\mathrm{H}_{2}$ redox potentials and to drive the water splitting reactions. We are mainly interested in the crystalline and the nano-crystalline materials. A general scheme to predictively tune materials band structure properties is presented below:

\section{How do we tune optical (band) gaps?}

1. By isovalent doping.

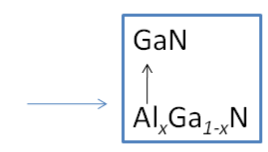

2. By passive co-doping.

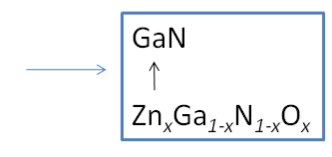

3. By predicting novel alloys Challenging for ab initio modeling!

\section{By reducing the dimensions.}

Nanostructures: Nanocrystal, etc.

Figure 3: Four strategies for improving properties of photoelectrode materials.

Two main research topics mainly targeting number 3 and 4 of the above scheme:

(i) Novel photo-catalysts prediction by mineral database searching: The discovery of efficient photo-catalysts is one of the grand challenges for energy conversion and storage. As naturally occurring materials do not fulfill all the required electronic criteria, these electronic requirements in materials are usually achieved by a band-engineering approach, where the electronic structures of the materials are engineered by selective doping. However, the introduction of impurities would create unwanted defect states in band gap, which would be detrimental to the transport properties of the host due to poor crystallinity. To-date shifting the optical absorption spectrum of a material to the visible region by doping-only process has not been successful in improving the photoconversion efficiency significantly.

Instead of following a simple band-engineering-only approach, we plan to follow a "natural selection" process followed by a selective band-engineering approach. As these materials were already formed in earth through natural selection and evolution processes 
over millions of years, these will be the ideal base candidates to design materials for any energy-related applications. Though these naturally occurring minerals by themselves may not be directly suitable for energy conversion, the knowledge of chemical compositions of these minerals may lead to a proper photo-conversion material. Their compositions and properties can easily be determined by theory calculations, and instead of doping a new alloy based on the selected mineral will be predicted. The drawback that exists in simple band-engineering-only processes, that is poor crystallinity, formation of charge traps etc., can be avoided in this mineral database searching method. These new structures then can be tested at the experimental lab. An extensive experimental feedback is needed for the successful prediction of new minerals. In this proposed research, we will also develop efficient selection algorithm for mineral database search. This work is currently going on in our group, and one graduate student is involved with this research.

(ii) Nano-crystalline photocatalysts: Understanding of electron photo-transition and transport is crucial for the efficient design of metal-oxide nano-crystalline photocatalysts. The current understanding at the "nano" level is not very clear, and leads to misleading assumptions to the photo-catalytic chemistry for these nano-crystals. First principle theories, such as DFT and time-dependent-DFT (TDDFT), are state of the art theoretical methods to shed light in these aspects. Several key issues remained challenging in metaloxide nano-crystals so far; some of them are: (i) identification of the fundamental gap and the actual optical gap, (ii) the nature of energy levels ("band") in the nano-crystals, (iii) the difference between the flat band potential and the band position in the "middle" of nanocrystals, (iv) transport of charge carriers after photo-excitation and (v) extraction or injection of electrons from or to the nano-crystals' surface.

Apart from the above challenging issues, metal-oxide nano-crystals can show unpredictable electronic behavior due to their unsaturated and charge uncompensated surface bonds. These charge unpassivated ionic bonds may attract different chemical species to passivate themselves, and hence the electronic properties of the nano-crystals may change significantly. Different sizes of nano-crystals can have different level of charge imbalances, so the compensation process may differ with different sizes, and results in unpredictable electronic behavior. Predictability is a key issue in manipulating the electronic properties of materials for various applications, such as photo-catalysis. We have recently shown that a unique set of self-passivated and charge compensated delafossite nano-crystals can be found which will have more predictive behavior. The existence of these highly stable nanocrystals needs to be explored in other metal oxides.

In addition, the optical absorption at nano-crystals may pose a different challenge. Recently, the optical absorptions at the nano-level for the delafossite metal-oxides were found to be zero for the first few excitation energies as calculated by TDDFT. This feature is very interesting, as this may suggest that the nano-level did not enhance the optical absorptions. However, this needs to be thoroughly studied for other metal oxides nanocrystals with various sizes as well. The research on metal oxide nanocrystals is also currently going on in Dr. Huda's group. However, no student or post-doc is involved in this project. 


\section{Task II: Characterization and Optimization of the Electrode-electrolyte Interface}

Focus: Gain atomistic insight into the properties of electrode-electrolyte interface in order to develop a corrosion mitigation strategy and to improve STH conversion efficiency.

As already discussed, PEC photoelectrodes presently demonstrate either high STH or high corrosion resistance, but not both simultaneously. The LLNL team is currently working with NREL to improve the corrosion resistance of $\mathrm{GaInP}_{2}$, which offers the highest STH conversion efficiency of any electrode material but suffers from severe lifetime issues. The approach uses first-principles molecular dynamics simulations to understand the structure and reactivity of the electrode-electrolyte interface, where corrosion and hydrogen evolution compete with one another. This information will be used to devise a strategy for improving device stability and performance.

LLNL's plan initially involves investigation of the $\mathrm{GaInP}_{2}(001)$ surface both with and without an electrolyte. This will be done in order to understand which microscopic interfacial properties are necessary to achieve high STH, and how these are affected by the presence of an electrolyte. We will then simulate and compare the in-situ XAS/XES data for our model systems with experimental results from the UNLV surface characterization team, which will allow us to verify which of our proposed atomistic mechanisms are indeed related to hydrogen evolution and corrosion. Simulation results will be compared with data from NREL's recent attempts at surface stabilization via nitrogen treatment. In doing so, we aim to extract a mechanism for atomistic surface stabilization and to develop an improved corrosion mitigation strategy for $\mathrm{GaInP}_{2}$. It is expected that these efforts will interface closely with and provide feedback to the rational materials design effort (Task I), particularly given that $\mathrm{Dr}$. Wei is recognized as one of the leading experts on $\mathrm{GaInP}_{2} \cdot{ }^{34-37}$
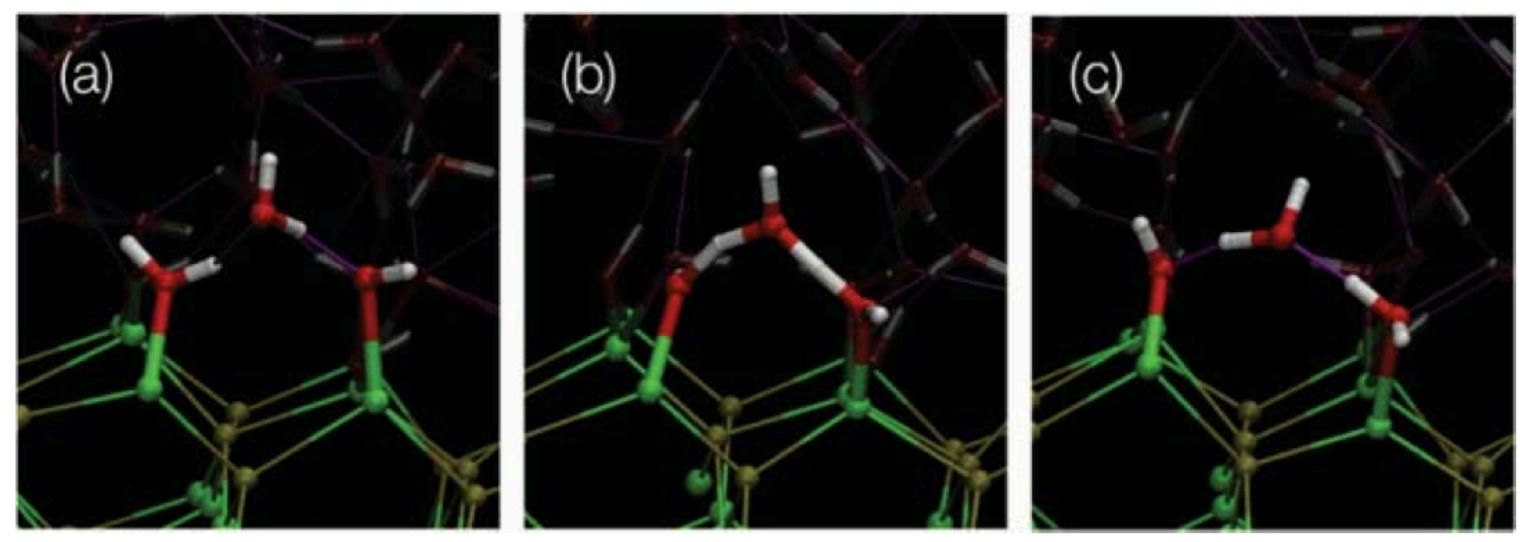

Figure 4: Proton transfer across a Grotthuss chain involving an adsorbed water molecule, a water molecule in solution, and an atop $\mathrm{OH}$ group on $\mathrm{InP}(001)$. Taken from a dynamics simulation of an oxygen-rich InP(001) surface in contact with water. Color scheme: $\mathrm{H}=$ white, $\mathrm{O}=$ red, In=green, $\mathrm{P}=$ gold.

To date, we have completed and published studies on morphologies of GaP(001) and InP(001) surfaces, including various types of surface oxide and hydroxide structures 
that form natively at the interface with water. ${ }^{29-33}$ We were able to identify certain structural motifs that have a significant impact on the interfacial properties and surface stability. For instance, surface hydroxylation was found to induce unusually strong hydrogen bonding and fast proton shuttling at the surface (see Fig. 5). In addition, surface oxidation can lead to reduced hole mobility, which may contribute to the observed photocorrosion of $\mathrm{GaInP}_{2}$ photoelectrodes. This hypothesis is currently being investigated experimentally using advanced in situ characterization techniques.

We are currently working on four additional subjects (detailed below), which we expect to continue through the remainder of FY12 and FY13: first, characterization of the $\mathrm{GaInP}_{2}$ (001)-water interface with and without surface oxides and hydroxides; second, the effect of substitutional impurities (e.g., nitrogen) on the electronic and chemical properties of the III-V/water interface; third, XAS/XES simulations of the aforementioned surfaces and interfaces; and fourth, development of a simple model to analyze catalytic reaction mechanisms in a computationally efficient manner.

i) Simulation of the GaInP $\underline{2}_{2}$ (001)-water interface: The first subtask involves the application of previously established static and dynamic methods for GaP(001) and $\operatorname{InP}(001)$ to the alloy material $\mathrm{GaInP}_{2}$ (001). In addition to extracting structural motifs that can be used in subsequent models, we will assess the role of alloy structure on the chemical properties of the interface. Simulations will be done with and without additional surface contaminants.

ii) Investigation of subsitutional impurities: In FY12, T. Deutsch et al. (NREL) discovered that a specific type of nitrogen incorporation to $\mathrm{GaInP}_{2}$ electrode may improve the durability significantly with an acceptably minimal impact on STH efficiency. For our second subtask, we intend to investigate in detail the effect of nitrogen doping on surface corrosion resistance in order to gain microscopic insight on the atomistic surface stabilization mechanism.

iii) Simulation of XAS/XES spectra: For our third subtask, we plan to simulate X-ray absorption (XAS) and emission (XES) spectra of reference model surfaces. These will be done on clean surfaces, as well as oxygen- and hydroxyl-contaminated surfaces, which are better approximations to the electrode structure when immersed in electrolyte. The goal of this stage is to establish and validate accurate models for the precursory interfacial structure present prior to illumination. For this part of research activity, the LLNL team is collaborating with Dr. Prendergast at LBNL (Computational Spectroscopy Group of The Molecular Foundry). Notably, optimized computational procedures for simulating the $\mathrm{P}-\mathrm{L}_{2,3}$ edge XAS/XES of GaInP 2 have already been established and tested (see Fig. 5).

Pending additional funding, our eventual plan is to use our simulation results to analyze the in situ and ex situ experimental data generated at later stages of the reaction cycle, including changes in the surface states, the generation of reaction intermediates, and the identification of final product species. From these, we will be able to more fully reconstruct the competing candidate reaction pathways. 
GaP

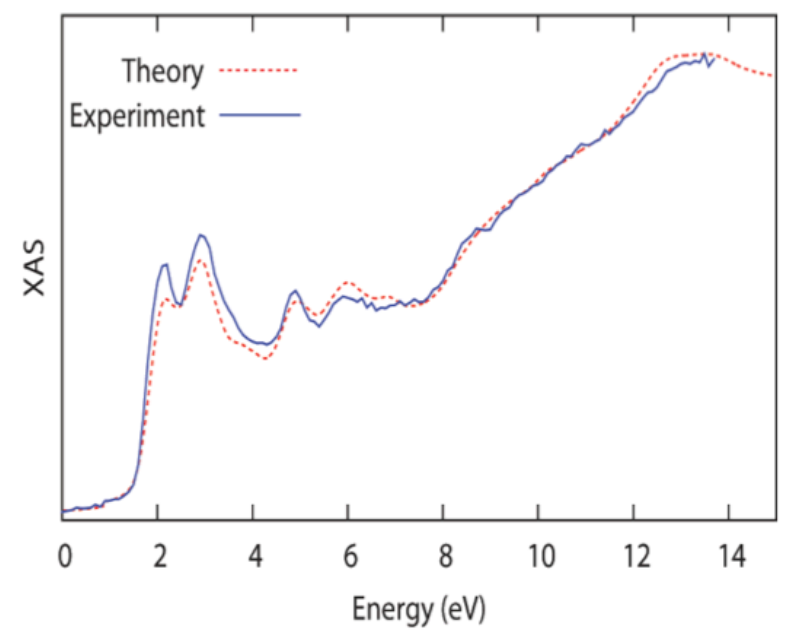

$\ln P$

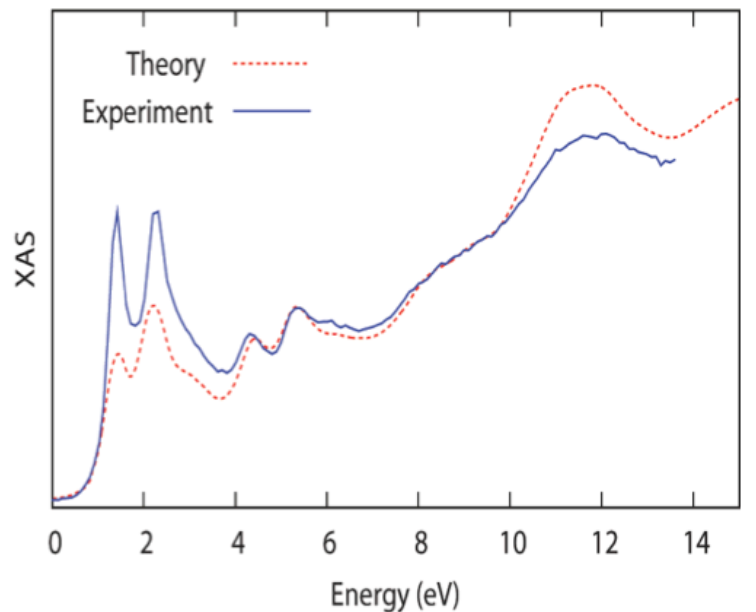

Figure 5: Calculated and experimental P-L2,3 edge XAS of GaP (left) and InP (right) in their bulk phases. From 2012 AMR presentation.

In addition, experimental characterization of actual GaInP 2 electrodes by the UNLV team found a $>1 \mathrm{~nm}$ native oxide, meaning the interface might better be modeled as indium/gallium oxide in contact with water. If resources permit, we would like to further develop and investigate such models, including the generation of corresponding computational spectra to compare with the UNLV/NREL results. These could be particularly useful for detailing the atomistic corrosion mechanism, as well as its relationship with the hydrogen evolution mechanism. An initial comparison of the experimental data with our current models should allow us to better assess the necessity of these additional spectra. If necessary, we will revise our model structures until we come to a satisfactory agreement between experiment and theory.

iv) Modeling of free-energy reaction profiles: For our fourth subtask, we aim to calculate the free-energy profiles and kinetic barriers of our proposed model reaction pathways to examine which ones will be relevant ones that are observed in the experiments (see Fig 6). For this stage, we may propose additional experiments that can be used to verify our analysis. Given the current workforce at both UNLV and LLNL, it is expected that this subtask will likely be conducted after FY13. Using such an approach, we can gain significant amount of information on how the relevant reactions take place in real devices without explicitly simulating the relatively slow hydrogen evolution and corrosion processes directly, which is not computationally feasible with current methods. In the future, this could be a valuable way of efficiently screening surface treatments to improve the catalytic performance of electrodes. 

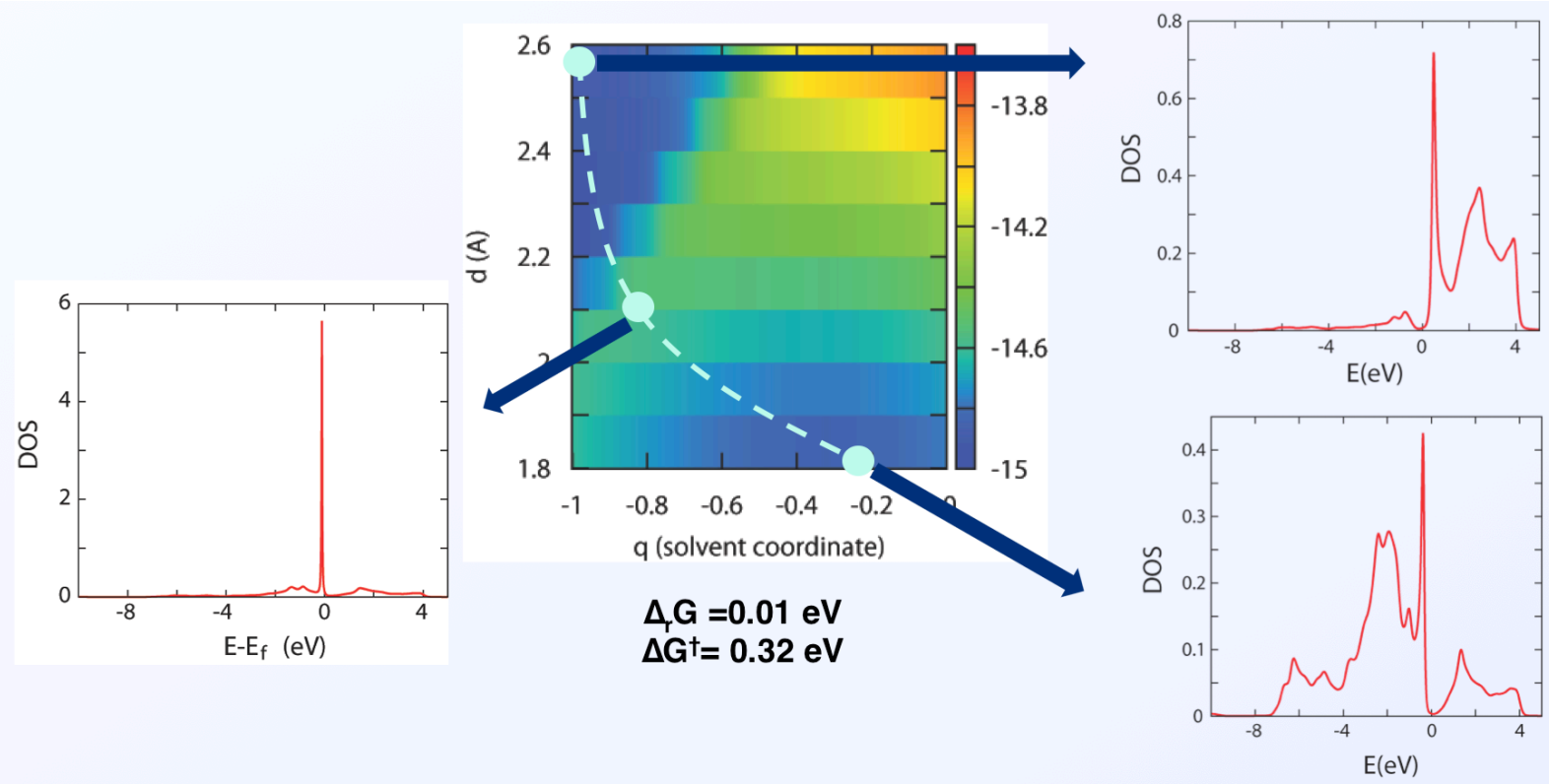

Figure 6: The free energy profile for the Volmer step of the hydrogen evolution reaction (proton adsorption/desorption from solution) at the $\mathrm{GaInP}_{2}$ (001)/water interface using the model Hamiltonian approach described for Pt in Phys. Rev. B 79,235436 (2009). Although application of a bias voltage changes the free energy profile to favor adsorption,

$\mathrm{GaInP}_{2}(001)$ retains a kinetic barrier that limits its intrinsic catalytic activity, due to lack of interaction between the surface atom and the hydrogen. The efficiency and predictive capability of this method makes it good for screening of potential catalysts and rationalizing why certain candidates should be excluded. From 2012 AMR presentation.

Finally, we note that we have gained relevant capability and additional qualified workforce without added financial burden by establishing a formal collaboration relation with an unfunded external collaborator, Dr. Otani of National Institute of Advanced Industrial Science and Technology (AIST), Japan, via the U.S.-Japan (DOE-METI) Clean Energy Technology Action Plan. Dr. Otani has co-developed an advanced method to implement a fixed bias potential within a first-principles simulation scheme. Using his Effective Screening Medium (ESM) Method, and we plan to investigate how the structural, electronic, and chemical properties of the water-electrode interface are impacted by the electric field that results from photoinduced carrier generation. This method brings us a significant step forward towards the direct comparison of our simulation results with experiments. It is worth pointing out that the ESM and the other methods described here are universal, allowing us to potentially consider other electrode materials, such as $\mathrm{MoS}_{2}$, which is one of the few materials that exhibit good catalytic activity for hydrogen evolution. If resources permit, this would be an excellent additional research avenue to explore in FY13.

\section{Summary}

In summary, we have outlined a twofold approach that uses advanced theoretical techniques to develop design and optimization strategies for efficient, durable PEC photoelectrodes. First, we use first-principles density functional theory calculations of the electronic, optical, and transport properties, as well as an investigation of the interplay of these properties in real devices, in order to efficiently screen candidate electrode materials. 
Second, we use first-principles molecular dynamics simulations and model free-energy reaction barrier calculations to examine the structure and chemistry of the surface, with the goal of improving device stability and performance. Together with the efforts of other members of the DOE/EERE Photoelectrochemical Hydrogen Production Working Group, the theory team's input should provide much-needed insight into how specific photoelectrode materials properties should be combined so as to engineer and optimize devices that meet the DOE market adoption targets. This work is supported by the DOE EERE Fuel Cell Technology Program, and partly performed under the auspices of the DOE by LLNL under Contract DE-AC52-07NA27344.

\section{Appendix: Summary of Required Properties}

1. Photoelectrode as a PV device must have a reasonable solar-to-electric conversion efficiency (15-20\% to achieve $10 \%$ overall STH).

a. Since the energy spectrum of sunlight is fixed, the band gap of photoelectrode cannot be too large $(<2.2 \mathrm{eV}$ for a single cell configuration. Could be smaller for a tandem cell/muti-junction cell).

b. The dipole matrix elements between $\mathrm{CB}$ and VB need to be large in order for photons to excite electrons to $\mathrm{CV}$ with high probability.

c. The transport property of CB and VB must be reasonably good (metal-oxide tend to have the transport problem).

d. One must minimize defects/disorder in order to minimize the exciton-hole recombination probability (alloying/doping could lead to disorder/defect).

2. In order to drive Hydrogen Evolution Reaction (HER) and Oxygen Evolution Reaction (OER), the conduction band (CB) and valence band (VB) of photoelectrode need to straddle redox potential for HER and OER. This imposes the constraint on the absolute positions of bottom of $\mathrm{CB}$ and the top of $\mathrm{VB}$, in addition to the minimum band gap of about $1.5 \mathrm{eV}$.

a. The band edge position could be influence by the other factors such as $\mathrm{pH}$, surface states, interface transport (interplay with co-catalyst)

3. Photoelectrode surface need to have a high catalytic activity for HER/OER

a. Low catalytic activity requires high over-potential (higher CB position for HER, lower VB for OER), and the offset to redox potential is lost as thermal energy.

b. Co-catalyst geometry configuration needs to be optimized so as not to interfere with photon collection

c. Co-catalyst need to be stable under operation condition (adhesion property) 


\section{References}

$1 \quad$ A. Fujishima and K. Honda, Nature 238, 37 (1972).

R. E. Rocheleau and E. L. Miller, Int J Hydrogen Energ 22, 771 (1997).

R. E. Rocheleau, E. L. Miller, and A. Misra, Energy \& Fuels 12, 3 (1998).

O. Khaselev and J. A. Turner, Science 280, 425 (1998).

"Hydrogen and Fuel Cells Program Plan", (Department of Energy, Washington D.C., 2011)

"Fuel Cell Technologies Program Multi-Year Research, Development and Demonstration Plan: 3.1 Hydrogen Production, 2011 Interim Update", (Department of Energy, Washington D.C., 2011) "Basic Research Needs for Solar Energy Etilization", (Department of Energy, Washington D.C., 2005)

"Hydrogen Posture Plan", (Department of Energy, Washington D.C., 2006)

"Basic Research Needs for the Hydrogen Economy", (Department of Energy, Washington D.C., 2004)

"National Hydrogen Energy Roadmap", (Department of Energy, Washington D.C., 2002)

"A National Vision of Ameriaca's Transition to A Hydrogen Economy -- To 2030 and Beyond", (Department of Energy, Washington D.C., 2002)

A. Walsh, J. L. F. Da Silva, Y. Yan, M. M. Al-Jassim, and S.-H. Wei, Phys Rev B 79 (2009). A. Walsh, et al., Energ Environ Sci 2, 774 (2009).

A. Walsh, Y. Yan, M. M. Al-Jassim, and S.-H. Wei, Journal of Physical Chemistry C 112, 12044 (2008).

A. Walsh, Y. Yan, M. N. Huda, M. M. Al-Jassim, and S.-H. Wei, Chem Mater 21, 547 (2009).

M. N. Huda, Y. F. Yan, S. H. Wei, and M. M. Al-Jassim, Phys Rev B 78 (2008).

M. N. Huda, Y. Yan, A. Walsh, S.-H. Wei, and M. M. Al-Jassim, Appl Phys Lett 94 (2009).

M. N. Huda, Y. Yan, A. Walsh, S.-H. Wei, and M. M. Al-Jassim, Phys Rev B 80 (2009).

M. N. Huda, Y. Yan, C.-Y. Moon, S.-H. Wei, and M. M. Al-Jassim, Phys Rev B 77 (2008).

W.-J. Yin, S.-H. Wei, M. M. Al-Jassim, and Y. Yan, Phys Rev Lett 106 (2011).

W.-J. Yin, S.-H. Wei, M. M. Al-Jassim, and Y. Yan, Appl Phys Lett 99 (2011).

W.-J. Yin, S.-H. Wei, M. M. Al-Jassim, J. Turner, and Y. Yan, Phys Rev B 83 (2011).

W.-J. Yin, H. Tang, S.-H. Wei, M. M. Al-Jassim, J. Turner, and Y. Yan, Phys Rev B 82 (2010).

M. N. Huda, Y. Yan, A. Walsh, S.-H. Wei, J. A. Turner, and M. M. Al-Jassim, in Solar Hydrogen and Nanotechnology V, edited by H. W. H. Idriss, 2010), Vol. 7770.

M. N. Huda, A. Walsh, Y. Yan, S.-H. Wei, and M. M. Al-Jassim, J Appl Phys 107 (2010).

C. Feng, W.-J. Yin, J. Nie, X. Zu, M. N. Huda, S.-H. Wei, M. M. Al-Jassim, J. A. Turner, and Y. Yan, Appl Phys Lett 100 (2012).

Y. Gai, J. Li, S.-S. Li, J.-B. Xia, and S.-H. Wei, Phys Rev Lett 102, 036402 (2009).

W.-J. Yin, S. Chen, J.-H. Yang, X.-G. Gong, Y. Yan, and S.-H. Wei, Appl Phys Lett 96, 221901 (2010). 
"Characterization and Optimization of PhotoelectrodeSurfaces for Solar-to-Chemical Fuel Conversion", (Department of Energy, Washington D.C., 2010) B. Wood, T. Ogitsu, and E. Schwegler, in SPIE Optics + Photonics 2010 (SPIE, San Diego, 2010).

B. C. Wood, T. Ogitsu, and E. Schwegler, Journal of Photonics for Energy 1, 016002 (2011).

B. C. Wood, T. Ogitsu, and E. Schwegler, The Journal of Chemical Physics 136, 064705 (2012).

"Characterization and Optimization of PhotoelectrodeSurfaces for Solar-to-Chemical Fuel Conversion", (Department of Energy, 2011)

S. H. Wei and A. Zunger, Appl Phys Lett 56, 662 (1990).

S.-H. Wei and A. Zunger, Phys Rev B 57, 8983 (1998).

A. Franceschetti, S.-H. Wei, and A. Zunger, Phys Rev B 52, 13992 (1995).

S.-H. Wei, D. B. Laks, and A. Zunger, Appl Phys Lett 62, 1937 (1993). 